\title{
El problema del cuidado desde la perspectiva de los niños(as): análisis crítico del discurso sobre el cuidado entre padres e hijos(as)*
}

\section{The Problem of Care from Children's Perspective: A Critical Discourse Analysis on Care between Parents and Children}

\author{
Paulina Chávez Ibarra ${ }^{a}$ \\ Universidad Academia de Humanismo Cristiano, Chile \\ ORCID: http://orcid.org/0000-0002-4910-6709 \\ Ana Vergara del Solar \\ Universidad Diego Portales, Chile \\ ORCID: http://orcid.org/0000-0001-7823-3769
}

\footnotetext{
a Autora de correspondencia. Correo electrónico: pchavezi@docentes.academia.cl
}

Para citar este artículo: Chávez, P. \& Vergara del Solar, S. (2018). El problema del cuidado desde la perspectiva de los niños(as): análisis crítico del discurso sobre el cuidado entre padres e hijos(as). Universitas Psychologica, 17(3), 1-11. https://doi.org/ 10.11144/Javeriana.upsy17-3.pcpn

\section{RESUMEN}

La moderna construcción social de la infancia, enfatiza su dependencia, fragilidad, y recepción pasiva y unilateral del cuidado adulto, invisibilizando el aporte que los niños(as) realizan al trabajo de cuidado, en los distintos escenarios cotidianos de los cuales participan. Este artículo expone los principales hallazgos de una investigación empírica basada en una perspectiva discursiva crítica, cuyo objetivo consistió en conocer los discursos que niños(as) de distintos estratos socioeconómicos de Santiago de Chile, construyen sobre las relaciones entre padres e hijos(as), centrándose en el trabajo cotidiano de cuidado, entendido como una dimensión constitutiva de dicha relación. Sus discursos dan cuenta de su agencia y competencia moral, evidenciando que las relaciones familiares de cuidado se sostienen en una red compleja y sutil de interdependencias. Palabras clave

infancia; relaciones padres-hijos; prácticas de cuidado; análisis crítico del discurso; psicología crítica; nuevos estudios sociales de la infancia.

\footnotetext{
ABSTRACT

Social construction of modern childhood emphasizes its dependence, fragility, and passive reception of adult care, making the contribution of children to care work in different everyday scenarios in which they participate, become invisible. This article presents the main findings of an empirical research, based on a critical discursive perspective whose objective was to know the discourses that children from different socioeconomic strata constructed on the parent-child relationships, focusing on the theme of daily care work, understood as a constitutive dimension of this relationship. Their discourses reflect their agency and moral competence, showing that family care relationships are held in a complex and subtle network of interdependencies.

Keywords

childhood; parent-child relationships; care practices; critical discourse analysis; critical psychology; chilhood studies.
} 
En coherencia con la lógica evolutiva del progreso, propia del imaginario moderno, la infancia ha sido socialmente construida como momento o etapa fundacional más cercana a la naturaleza que a la cultura, que requiere un arduo trabajo de cuidado, educación y control, para ir progresivamente ascendiendo "de inmadurez a madurez, de incapacidad a capacidad, de irresponsabilidad a responsabilidad" (Qvortrup, 1992, p.177), hasta llegar a la condición ideal de sujeto racional y autónomo, capaz de formar parte de la construcción del mundo social, equivalente al mundo adulto.

Si bien a partir de la segunda mitad del siglo XX, importantes transformaciones socioculturales han aportado al reconocimiento y valoración social de los niños(as) como 'sujetos de derechos', expandiendo los discursos y ámbitos en los que se legitima su competencia, agencia y participación, dichas transformaciones coexisten con una serie contradicciones en lo que respecta a los espacios efectivos de participación que nuestra sociedad les ofrece y a la genuina consideración de su condición de actores sociales, cuyos discursos y experiencias, también forman parte de la configuración del mundo social.

Aunque las distintas representaciones históricas de la infancia moderna son múltiples

y contradictorias ${ }^{i}$, es posible observar un predominio de modelos unidireccionales de socialización que enfatizan la subordinación y dependencia de los niños(as) en relación con los adultos, así como el cuidado, protección y autoridad de adultos a niños(as), posicionando a estos últimos del lado del no saber, la fragilidad y la incompetencia moral (Giberti, 1997). Así, nuestras sociedades occidentales han pensado la infancia y legitimado el rol adulto con base a relaciones asimétricas e irreversibles de cuidado entre adultos 'protectores' -cuidadores, responsables, activos- y niños(as) 'protegidos' pasivos, vulnerables, inexpertos-, cuestión que constituye una potente 'base ideológica' sobre la que adultos y niños(as) construyen sus relaciones (Mayall, 2002). Esto no implica negar el hecho biológico de la 'inmadurez', dependencia o necesidad de cuidado de los niños(as), sino que apunta más bien, a evidenciar que estas representaciones que contraponen dependencia y autonomía, así como las prácticas adultas que de ahí se desprenden, han operado históricamente negando sus voces, socavando su agencia en la producción, reproducción y transformación de las relaciones sociales en las cuales participan.

Por otra parte, la reflexión crítica sobre la problemática del cuidado se ha centrado principalmente en el mundo adulto, orientándose a dar cuenta de la dimensión social y política de desigualdades étnicas, de clase $y$, fundamentalmente de género -división del trabajo entre hombres y mujeres- (Comas d'Argemir, 2014). En el caso de los estudios sobre infancia, buena parte de ellos busca conocer las principales actividades domésticas o extra domésticas que los niños(as) realizan, en función de actualizar los diagnósticos sobre el problema social del trabajo infantil realizado en condiciones peligrosas o de explotación, para poder evaluar y diseñar políticas públicas orientadas a su erradicación (Macri, Ford, Berliner \& Molteni, 2005). En este escenario la comprensión de las significaciones que los propios niños(as) articulan sobre los espacios cotidianos de cuidado e interacción con el mundo adulto, aparece como una tarea relevante y todavía pendiente (Chávez, 2015).

Con el propósito de aportar a la reflexión crítica sobre la problemática del cuidado y visibilizar a los niños(as) como actores sociales que participan de la producción de discursos sociales sobre las relaciones familiares, el presente trabajo expone los principales resultados de una investigación empírica de carácter cualitativo, realizada entre los años 2010 y 2014. Su objetivo consistió en conocer los discursos que niños y niñas de 10 y 11 años, de distintos estratos socioeconómicos de Santiago de Chile, construyen sobre la infancia, la adultez y las relaciones establecidas con los adultos. El presente artículo se focaliza en la temática del trabajo cotidiano de cuidado, entendido como dimensión constitutiva de 
la relación intergeneracional entre padres e hijos(as). Teóricamente, esta investigación se basa en aportes de la psicología crítica, el campo interdisciplinar de los Nuevos Estudios Sociales de la Infancia (Childhood Studies) y el Análisis Crítico del Discurso (ACD) de Norman Fairclough $(1995,2003)$.

Hacia un abordaje crítico de la infancia y las prácticas de cuidado

En el contexto de una crítica al abordaje esencialista y universalizante de la infancia, a fines de los años 90' surge en el mundo anglosajón el campo interdisciplinario de los denominados Nuevos Estudios Sociales de la Infancia (Childhood Studies), que en la actualidad configuran un territorio amplio, heterogéneo e interdisciplinar, desarrollado tanto en Europa como en América Latina y Norteamérica (Qvortrup, Corsaro \& Honing, 2009). Este campo reúne diversas perspectivas críticas, que en el caso de la psicología, buscan deconstruir y problematizar las lecturas hegemónicas de la infancia, propias de la psicología evolutiva (Burman, 2012).

En este marco, la infancia es considerada como un objeto de estudio por derecho propio y no simplemente como variable a incluir en los estudios de familia: género, educación, o políticas públicas. No obstante, su análisis de comprensión como construcción social heterogénea y dinámica, incorpora sus estrechas articulaciones con la estructuración para las transformaciones sociohistóricas, culturales y políticas, de dimensiones como la clase social, el género y la etnicidad, entre otras. Asimismo, se distingue entre la infancia como categoría e institución social los niños(as) 'concretos' y particulares, en quienes se encarna esta formación social, institucional y discursiva de la infancia (Prout \& James, 1997). Por otra parte, se plantea que el estudio de la infancia, al igual que el género o la clase social, debe incorporar su dimensión relacional, esto es el marco de relaciones entre adultos y niños(as), entendidas como relaciones sociales, históricamente configuradas y trazadas por la desigualdad. De este modo, se entiende que las categorías generacionales 'adulto' y 'niño(a)' se articulan constitutivamente, son interdependientes e intrínsecamente relacionales (Mayall, 2002).

Una de las relaciones intergeneracionales social y subjetivamente más significativas para los niños(as), es la establecida con sus padres. En tanto institución social y construcción sociohistórica, atraviesan ésta relación por dimensiones de clase, género, étnicas, entre otras, operando tanto en un nivel macrosocial como cotidiano y microsocial, mediante la asignación de posiciones sociales a padres e hijos(as), en función de criterios etario-generacionales que determinan distintas experiencias y representaciones de lo que es y debe ser-un hijo(a), un padre/madre y las relaciones entre ellos. Esto se puede distinguir en los actores concretos (padres, hijos/as), cuyas prácticas así como relaciones cotidianas no sólo reproducen, sino que también transforman las instituciones de la parentalidad y la 'hijidad' ii . Desde esta perspectiva, lo que acontece entre padres e hijos(as) no responde simplemente a competencias o características individuales, particulares y 'propias' de adultos o niños(as) (Burman, 1998).

La relación social padres-hijos(as) se encuentra en estrecho vínculo con estructuras patriarcales de control que, bajo la lógica de la división sexual moderna del trabajo, recaen sobre el ámbito privado y público, distribuyendo diferencialmente a hombres y mujeres funciones o roles 'propios' del ejercicio parental. La moderna razón productivista contrapone producción (actividad, autonomía, esfera pública) a reproducción (pasividad, dependencia, esfera privada doméstica), dejando el trabajo de cuidado del lado de una domesticidad 'improductiva' en términos económicos, naturalizada como el espacio primario de lo femenino y lo materno.

Una mirada crítica a esta perspectiva es articulada a partir de los años 60' por conceptualizaciones feministas que incorporan la dimensión política del cuidado, denunciando 
cómo, la concepción mercantil del trabajo, invisibiliza el carácter productivo y valor social de las actividades domésticas y de cuidado realizada por las mujeres, lo que conlleva una "desigual distribución de recursos, derechos y deberes" (Legarreta, 2006, p.219). Asimismo, se critica la cultura del cuidado promovida por los marcos culturales y valóricos capitalistas, basada en pares dicotómicos y excluyentes (autonomía-dependencia, suficiencia-incompetencia, actividad-pasividad), los cuales se naturalizan como atributos individuales, reduciendo la dependencia a una condición individual estática, en lugar de reconocerla "como un cambiante resultado de procesos sociales” (Pérez, 2006, p.13).

Los aportes de las reflexiones sobre una 'ética del cuidado' (Gilligan, 1982), basada en el reconocimiento la valoración de la diferencia, y diversidad humanas, en contextos relacionales específicos e interdependientes de cuidado y responsabilidad, han permitido repensar las perspectivas universalistas y racionalistas de la ética, las cuales promueven un modelo tanto abstracto como excluyente de ser humano (adulto, varón, autónomo, independiente, occidental, de clase media, etc.), obturando la posibilidad de incorporar la experiencia y agencia de aquellos sujetos que, como los niños(as), no se ajustan a estas definiciones.

Si bien los aportes y debates feministas sobre el cuidado han tenido una importante recepción en los estudios críticos de la infancia sí como de la familia, la investigación de la perspectiva de los propios niños(as) en relación al trabajo de cuidado que estos realizan en los distintos escenarios cotidianos de los cuales participan (familia, escuela), es aún escasa (Luttrell, 2007, 2012). Por otra parte, en los estudios que incorporan la dimensión intergeneracional del cuidado todavía predomina una imagen irreversible y unilateral de las prácticas de cuidado adulto, en el marco de relaciones que se piensan como estáticas y de dirección fija: el cuidador adulto sólo cuida mientras el niño(a) objeto del cuidado solamente recibe dichos cuidados (Katz, 2013). Lo anterior impide pensar a los niños(as) como sujetos sociales que aportan activamente al trabajo de cuidado de otros y de sí mismos, ignorando o minimizando su participación en las complejas dinámicas de (re)producción de la vida familiar, en el marco de procesos de influencia recíproca entre padres e hijos(as).

La comprensión crítica del trabajo cotidiano de los niños(as) en distintas tareas de cuidado y organización del ámbito doméstico, demanda pensar la atención a los niños(as) como un trabajo permanente de creación, sostén material, afectivo e intersubjetivo de la vida cotidiana, sobre el marco de una condición humana vulnerable, (inter)dependiente y finita, en la que "todos somos a la vez [en distintos momentos, posiciones, formas y grados] cuidadores y objeto de cuidados" (Pérez, 2006, p.12). Lo anterior dista de la nocio\#n de trabajo propia de los discursos econo\#micos androcéntricos y adultocéntricos, que equiparan trabajo a empleo y significan el trabajo infantil como aquello que los niños, en tanto 'no adultos', no deben hacer, asociándolo básicamente a condiciones de pobreza, abuso y negligencia parental.

Finalmente, desde la perspectiva discursiva crítica adoptada en este estudio, los discursos de los niños(as), en tanto actores sociales, se piensan en el marco de prácticas y condiciones sociohistóricas que determinan las particulares propiedades y texturas de dichos discursos. En este sentido, resulta fundamental considerar el contexto actual de crisis de las formas en que nuestras sociedades gestionan y garantizan el trabajo de cuidado. La negación de la responsabilidad social en la sostenibilidad de la vida al igual que la generalizada precarización neoliberal de la vida en común. Todo esto configura un escenario actual en el que el cuidado familiar se ha sobrecargado de imperativos y exigencias, que sumergen el ejercicio de la parentalidad "en un ambiente [social] de responsabilidad y culpabilidad individual" (Böök \& Perälä-Littunen, 2008, p.76). 


\section{Método}

Los resultados expuestos en este trabajo son fruto de una investigación de carácter cualitativo, basada en una perspectiva hermenéuticointerpretativa, que adhiere a la propuesta teórico metodológica del Análisis Crítico del Discurso (ACD) sugerida por Norman Fairclough (1995, 2003). Para el ACD, el discurso se entiende como práctica social de producción intersubjetiva de significado, inscrita en el marco de contextos sociales, históricos, culturales e institucionales más amplios. En coherencia con esto, los relatos de los niños(as) se comprenden como discursos sociales y culturalmente construidos, que participan activamente en la producción, reproducción y eventual transformación de las relaciones sociales desiguales establecidas entre niños(as) y adultos (Alldred \& Burman, 2006).

Se seleccionaron intencionalmente un total de 48 sujetos (24 niños y 24 niñas), de tres estratos socioeconómicos (medio alto, medio y bajo), entre 10 y 11 años de edad, considerando que esta edad responde al momento límite de la autodefinición identitaria como 'niños(as)' (en edades superiores suelen alternar entre definirse como niños(as), adolescentes o jóvenes). El acceso a los niños(as) se realizó a través de establecimientos educacionales mixtos y laicos de distintas comunas de Santiago de Chile, que fueron seleccionados de acuerdo a los porcentajes de habitantes pertenecientes al estrato socioeconómico respectivo. En cuanto a las consideraciones éticas, se trabajó sólo con niños(as) interesados en participar de la investigación, autorizados por sus padres o adultos a cargo, utilizando protocolos de asentimiento - para los niños(as)y de consentimiento informado -para los padres-.Como técnica de producción de información, se llevaron a cabo entrevistas grupales no directivas. El corpus textual del presente análisis, estuvo constituido por 12 entrevistas grupales. En cada estrato participaron 16 niños(as) distribuidos en dos grupos: varones y niñas, cada uno de 8 integrantes. Con cada grupo se realizaron dos entrevistas. El proceso de análisis e interpretación del material incluyó las modalidades de caracterización o "calificación discursiva" de los significantes en estudio (qué es, cómo es, cómo son los actores que en ella participan, etc.) y la consideración del uso de particulares recursos o estrategias lingüístico-retóricas utilizadas por los sujetos para legitimar las representaciones producidas (naturalización, omisión, etc.). Asimismo, la interpretación incorporó elementos del contexto social, institucional y cultural más amplio, en el cual se inscriben los discursos ${ }^{\mathrm{iii}}$.

\section{Resultados}

En los discursos de los niños(as) el cuidado aparece como un elemento central para referirse tanto al ejercicio adulto de la parentalidad, como a las formas en que ellos, desde su condición de hijos(as), participan en las prácticas y dinámicas de la vida familiar.

Como agentes de cuidado, los padres resultan figuras legitimadas en su condición de adultos, al ser sujetos protectores, participanndo del difícil mundo del trabajo asalariado y tomando decisiones relevantes para la familia. Los niños(as) perciben con mucha claridad el gran esfuerzo cotidiano de sus padres para cuidarlos, en el contexto de un orden social neoliberal, en el que el trabajo cotidiano de cuidado y subsistencia familiar (el 'ganarse' la vida) recae en la eficacia de estrategias individuales y adquiere un formato predominantemente 'sacrificial', sobre todo en el caso de las madres. Asimismo, el cuidado parental constituye un referente discursivo que permite trazar una frontera generacional entre niños(as) y adultos, en la medida en que la responsabilidad se distribuye de manera diferente: los padres no sólo son responsables de sí mismos, sino también son los principales responsables de sus hijos(as).

"(...) cuando uno es grande, tiene que trabajar, cuidar a su hijo y para tener una casa hay que pagar la luz, hay que pagar el agua, hay que pagar todo...ganarse la vida" (Niños varones, estrato medio, primera entrevista, p. 4, octubre 4 de 2010). 
La noción de 'crianza' es el significante específico que los niños(as) ofrecen para referir las prácticas de cuidado parental, como lazo que une a los adultos en tanto padres -los que crían-, con los niños(as) en tanto hijos(as) -los que son criados-. Este significante sintetiza el arduo trabajo parental de enseñanza, transmisión de valores, cuidado y sostén económico de padres a hijos(as). Una 'buena' crianza garantizaría el resguardo y mantención del tiempo de la infancia, posibilitando que los niños(as), como receptores de las prácticas de crianza ('te crían', 'te llevan'), se mantengan dentro de los límites del territorio de una 'buena' infancia ${ }^{\text {iv }}$. En otras palabras, el cuidado adulto es lo que permite sostener y resguardar que un niño(a) pueda ser niño(a) todo el tiempo que 'corresponde': jugar, divertirse, no sufrir, no trabajar, tener bienestar económico, cariño, enseñanza, en suma, que sea feliz. Sin este cuidado, simplemente no sería posible 'tener' una infancia (lo cual correspondería al caso de los niños(as) que 'se cuidan solos').

"Es que si te crían bien, si te crían en una casa con todo lo necesario, con amor y con cariño y te llevan a un colegio que te enseña bien y todo, tú puedes jugar y puedes divertirte" (Niñas, estrato alto, segunda entrevista, p.8, agosto 31 de 2012).

"También los niños que pueden ser ricos y los papás no les ponen mucha atención y los niños se cuidan solos, no juegan, trabajan y así van creciendo sin tener infancia" (Niños varones, estrato alto, primera entrevista, p. 9, julio 25 de 2012).

Como dimensión afectiva y moral de la relación padres-hijos(as), el cuidado constituye un núcleo semántico fundamental en la definición y valoración del trabajo adulto ininterrumpido de generar y mantener un 'soporte' de la infancia, como territorio idealizado, protegido, especializado y segregado del mundo adulto ${ }^{\mathrm{v}}$, susceptible de ser habitado por los niños(as). Sus relatos, transmiten la noción de una infancia que se juega y depende radicalmente de los padres nucleares y de las relaciones que ellos establecen con los niños(as): no hay Estado, no hay familia extensa, no hay soporte social, no hay nada más que el esfuerzo y sacrificio (o negligencia) adulta individual.

Junto a esta imagen unidireccional y dicotómica (dependencia/autonomía) del cuidado realizado por los padres, coexisten discursos sobre sus propias prácticas en el hogar, que los visibilizan como actores que asumen responsabilidades y colaboran en la gestión cotidiana de la vida familiar. Estos discursos evidencian una importante competencia moral en el análisis de las relaciones establecidas con sus padres, asociada a un profundo sentimiento de gratitud y preocupación por su bienestar y por la retribución de cuidados en la familia, sobre todo, en el caso de las niñas. En el caso de los varones, ellos se sitúan fundamentalmente como receptores de cuidado familiar y parental, tematizando en menor medida los aspectos relativos a su agencia en este campo relacional. Asimismo, sus discursos tienden, en mayor medida a la reproducción de ciertos estereotipos de género. Si bien esto podría relacionarse con una reproducción discursiva de desigualdades sociales de género en la distribución, percepción y valoración del trabajo de cuidado doméstico, cabe señalar que algunos estudios muestran que cuando a los niños varones se les permite explayarse sobre otros espacios relacionales (escuela, relaciones entre pares), la problemática de la reciprocidad del cuidado, la responsabilidad, protección y apoyo emocional a otros, aparece teñida de muchos más matices y distinciones (Luttrell, 2012). Por ello, resulta importante no clausurar la interpretación de sus discursos sobre el cuidado, basándose sólo en lo expresado a propósito de las relaciones establecidas con los adultos en contextos familiares.

En el caso particular de las niñas, se observa un esfuerzo por retribuir el trabajo de cuidado parental y mantener cierta justicia en la distribución e intercambio familiar, a través de la realización de pequeñas acciones cotidianas silenciosas e invisibles- tales como: cumplir con sus deberes escolares, cuidar hermanos menores, realizar algunas tareas domésticas, obedecer las normas -aunque no siempre concuerden con su sentido-, esforzarse por manejar sus necesidades de consumo con austeridad, entre otras. Esto 
da cuenta que agencia y dependencia no están necesariamente en contradicción cuando se comprenden desde la óptica del cuidado y la interdependencia.

" $\mathrm{N}_{1}$ : Sí. Ellos no saben que nosotros nos esforzamos para que ellos estén bien/ $\mathrm{N}_{2}$ : Entonces, después les devolvemos el favor haciendo cosas/ Investigadora: Cosas, icómo? / $\mathrm{N}_{2}$ : Lavándole el auto, poniéndole plata en la cartera, lavándole la ropa, cocinando con ellos, lavar la loza/ N 3 : Acompañándolos" (Niñas, estrato alto, primera entrevista, p. 20, junio 25 de 2012).

Entre las acciones que las niñas realizan para retribuir el esfuerzo y colaborar con el trabajo parental, el 'portarse bien' -obedecer, tener buen trato con los padres- constituye una de las más importantes.

"N: Yo creo que lo mejor es portándose bien, porque los papás lo que quieren es que lo que ellos nos dan a nosotros, nosotros le demos un mejor trato, que nos portemos bien y eso es lo que les da mejor alegría que portarnos mal y que ellos sufren porque nos portamos mal/ Investigadora: $\mathrm{O}$ sea, que cuando ustedes se portan mal como que están así un poco como.../ $\mathrm{N}$ : En contra de ellos" (Niñas, estrato medio, primera entrevista, p. 22, noviembre 8 de 2010).

La valoración negativa de la desobediencia se inscribe en un discurso sobre la preocupación afectiva por el otro, más que en un discurso normativo de la 'distribución' de deberes y derechos. Es decir, las prácticas de obediencia asociadas a un 'buen trato' del otro adulto se orientan a resguardar el orden recíproco de los afectos (dar alegría, evitar el sufrimiento del otro). Esto da cuenta de que la solidaridad y preocupación afectiva aparecen como referentes centrales de sus concepciones morales de la condición de hijo(a). Asimismo, las citas anteriores ilustran tanto actividades efectivas de cuidado (portarse bien, colaborar en las tareas del hogar), centradas en las necesidades de los padres, como una preocupación o cuidado emotivo ('preocuparse por'), que expresa sentimientos asociados a una ética que reconoce las necesidades del otro y busca responderlas, elementos que han sido destacados por los estudios feministas como dimensiones centrales del cuidado (Cerri \& Alamillo-Martínez, 2012). Esto contraviene la imagen de la obediencia como parte de lo que corresponde 'naturalmente' a los niños(as) en el marco normativo del orden asimétrico de las generaciones y pone en juego una lógica de la reciprocidad, que se desmarca del cálculo estratégico (costo-beneficio, obedienciapremio/desobediencia-sanción), con el que suele interpretarse el ajuste de los niños(as) a las demandas adultas. Por otra parte, las niñas también reconocen, valoran y se preocupan por el arduo trabajo que los padres realizan para mantener las condiciones económicas y calidad de vida de las familias, evidenciando un conocimiento bastante detallado del consumo y gasto familiar. En este contexto, narran una serie de acciones de retribución, compensación y colaboración, orientadas a mantener los equilibrios y ajustes familiares.

\section{"N: (...) a veces mi mamá me compra cosas y yo las voy a pagar, entonces, mi mamá no lo acepta, entonces, yo pienso que mi mamá dice, así como que piensa, 'ipa' qué le compro esto?, pero ella va a ser feliz con esto', entonces, a veces como ella no me deja, yo le pido la llave a mi papá de mi alcancía, entonces, saco dos lucas ${ }^{\text {vi }}$ más de lo que mi mamá me pasó y yo las meto en su cartera" (Niñas, estrato alto, primera entrevista, p. 19, junio 25 de 2012).}

Este acercamiento a la problemática del dinero y el consumo, dista mucho de la imagen mediática de un niño(a) 'consumista', frívolo, inmaduro y desmesurado, centrado en la satisfacción de sus propias necesidades y deseos, cuya participación en la economía familiar se reduce a ser una potente influencia en las opciones de consumo de sus padres. Esto constituye 'contra-narrativas' vii a la imagen dominante de un agente cuya racionalidad se centra en el cálculo de costos y beneficios individuales, expresando una racionalidad moral orientada al cuidado de aquellos que cuidan (sus padres). A través de estos gestos cotidianos de retribución, las niñas no sólo expresan gratitud, sino que también facilitan que sus padres 
sostengan la ardua tarea social de posicionarse como 'proveedores' y cuidadores de sus hijos, evidenciando un conocimiento de las sutilezas que este posicionamiento implica.

El cuidado familiar privatizado y el constante trabajo de una parentalidad 'intensiva' $y$ 'sacrificial' (Lee, Bristow, Faircloth \& Macvarish, 2014), propios de nuestras actuales sociedades, también genera en los niños(as) sentimientos de malestar y tristeza. Así, tensionando la imagen de la infancia como tiempo ideal de felicidad, cuidado y ausencia de responsabilidades sociales, sus discursos dan cuenta de que la posición de 'ser cuidado', 'sostenido' y 'mantenido' emocional y materialmente, no resulta un lugar tan cómodo, fácil y placentero de habitar.

“(...) Sí, porque me da pena mi mamá o mi papá cuando ellos trabajan y todo eso, yo también me trato de esforzar para que ellos también puedan comprarse lo que ellos quieren" (Niñas, estrato medio, primera entrevista, p. 19 , noviembre 8 de 2010).

Ahora bien, un aspecto central de los relatos de las niñas de los distintos estratos, comprende la complejidad y pluralidad del sentido del cuidado, cuando se intersecta dimensión de género con problemática etaria. De este modo, la centralidad del cuidado en las construcciones identitarias femeninas, como expresión de la reproducción de las desigualdades de género propias de la moderna división sexual del trabajo, adquiere otro matiz incorporando una perspectiva de las niñas, en la medida que su preocupación moral por el cuidado y reciprocidad en las relaciones establecidas con sus padres, contraviene las visiones hegemónicas idealizadas de la infancia y puede verse como expresión de su capacidad de agencia y competencia moral como sujetos sociales. Ambas dimensiones, coexisten de maneras complejas, planteando importantes desafíos al análisis crítico de los distintos ejes que estructuran el orden social las cuales se expresan, recrean y subvierten, en los discursos de los sujetos. En esta misma línea, es posible leer esta suerte de carácter 'invisible' para los adultos de los esfuerzos y acciones cotidianas de retribución y cuidado realizadas por las niñas, quienes lejos de articular una queja hacia los padres que no ven este trabajo, le otorgan cierto valor a su carácter silencioso y altruista. En clave de género, esto podría interpretarse como signo de la temprana 'eficacia' de la transmisión de valores culturales hegemónicos, que asocian el cuidado a la 'naturaleza' afectiva femenina, orientada al sostén abnegado y silencioso de otros. Sin embargo, sus relatos alteran las representaciones de los niños(as) como seres pasivos, sometidos a un intercambio unidireccional -meros receptores de cuidado- y contravienen una cultura enfocada en reforzar valores competitivos, individualistas e instrumentales dentro de las relaciones sociales, dando cuenta de su participación en una economía relacional 'alternativa' dentro de sus familias.

En el caso específico de las niñas del estrato bajo, esta invisibilidad o naturalización del cuidado femenino podría asociarse a la significación que ofrecen de su participación en la realización de tareas cotidianas (aseo del hogar, cuidado de niños(as) pequeños, etc.) viii . Para ellas, estas tareas no constituyen una carga o simple colaboración voluntaria en el hogar, sino que forman parte de las responsabilidades que van asumiendo al crecer, representándose además como instancias de valioso aprendizaje del quehacer 'femenino' de cuidado.

"Y después nació mi otra primita y cuando la llevan pa' la casa yo la cuido, yo sé cómo cambiarla, cómo vestirla, se ya cómo hacer eso porque cuidando mi otra prima yo ya aprendí todo ese proceso" (Niñas, estrato bajo, segunda entrevista, p. 7, junio 6 de 2012).

Junto a la relevancia de una lectura crítica de la naturalización del cuidado como trabajo y capacidad 'propiamente' femenina, así como de la desigualdad estructural determinada por la pertenencia de estas niñas a un estrato socioeconómico bajo, que redobla las injusticias en la distribución social del trabajo cotidiano de cuidado, la dimensión etaria introduce matices importantes. Por una parte, en contraposición al mito liberal del sujeto autónomo, sus discursos evidencian que "el sujeto femenino del trabajo doméstico no [es] un sujeto individual, sino 
colectivo" (Pérez, 2006, p. 19), cuestión que no sólo da cuenta de una 'ética del cuidado' y preocupación del otro -al modo de la ética feminista-, sino también de una ética de la reciprocidad o del cuidado con otros, que se juega en un escenario moral concreto de responsabilidad, resistencia y enfrentamiento colectivo de las situaciones que impone una realidad hostil, mostrando así una mayor interdependencia entre adultos y niños(as) en el enfrentamiento de estas necesidades cotidianas. Por otra parte, si bien es cierto que el cuidado de otros niños(as) menores -hermanos(as), primos(as) - da cuenta que las niñas de este estrato asumen trabajos que no tienen una cobertura social adecuada, no es menos cierto que estas actividades de cuidado, asumidas con responsabilidad y compromiso afectivo, reivindican la agencia y competencia moral de las niñas en sus vidas presentes, subvirtiendo la imagen convencional de los niños(as) como sujetos carentes de responsabilidades, destinados a alcanzar un estatus moral reconocible sólo al llegar a la edad adulta. Este énfasis en la orientación hacia el futuro en el acercamiento a la infancia, repercute en aquello que los niños(as) pueden y deben 'llegar a ser, dificulta la comprensión de la complejidad de sus contextos presentes, en el marco del cual los niños(as) no operan como un puro 'devenir'. En un sentido restringido, las actividades que los niños(as) realizan en la escuela o el hogar, son significadas sólo como un 'capital' que llegará a ser parte de sus vidas futuras y no como un trabajo que configura y da sentido a sus vidas presentes, formando parte de su agencia. Este predominio de la 'futuridad' como tiempo en el que se representa a la infancia (Cockburn, 2005), junto con una imagen restringida de la noción de trabajo, conlleva que el trabajo cotidiano realizado por los niños(as) sea considerado como una suerte de 'como sî', esto es, como una simple preparación para el 'verdadero' trabajo (adulto) reforzando la segregación socioespacial y simbólica de los territorios de la infancia y la adultez. En contraposición a estas ideas, la manera como los niños(as) enfrentan y significan el trabajo de cuidado de otros y el valioso aporte material y afectivo que esto significa para sus familias, resultan evidencias suficientes para considerarlo un trabajo 'en serio', que forma parte importante del cuidado de sus vidas y las de sus familias.

\section{Conclusión}

A través de esta breve exposición de algunos tópicos discursivos referidos a la problemática del cuidado entre padres e hijos(as), esta investigación pretende ilustrar cómo los niños(as) articulan una serie de representaciones que dan cuenta de su agencia y competencia moral, constituyendo 'contra-narrativas' a los discursos modernos sobre el lugar de la infancia en el orden generacional de las familias. Sus discursos permiten comprender cómo las relaciones de cuidado y soporte de una infancia protegida, no se agotan en una dimensión de exclusiva dependencia niño(a)-adulto, sino que se sostienen en una red compleja y sutil de interrelaciones o interdependencias. Asimismo, evidencian la multidimensionalidad de la problemática del cuidado y la complejidad de su interpretación, cuando ésta incorpora la perspectiva de sujetos socialmente invisibilizados en su agencia en función de su condición etaria. Esto plantea importantes y nuevos desafíos al trabajo crítico de deconstrucción de las coordenadas tradicionales independencia/ dependencia, con las que se ha pensado la problemática del cuidado y el sujeto moderno, dejando a los niños(as) del lado de la dependencia y la pasividad. En este contexto, repensar la infancia y las relaciones que establece con el mundo adulto, bajo modelos que incorporen la interdependencia y la posibilidad de agencia de los niños(as), resulta una tarea central para interrogar las coordenadas teóricas y metodológicas con las que tradicionalmente los adultos se han acercado a la niñez. 


\section{Agradecimientos}

Esta investigación se desarrolló en marco de los proyectos Fondecyt Regular № 1120025 y $\mathrm{N}^{\circ} 1160650$.

\section{Referencias}

Alldred, P. \& Burman, E. (2006). Analysing children's accounts using discourse analysis. En S. Greene \& D. Hogan (Eds), Researching children's experience: Approaches and methods (pp.175-198). London: Sage.

Böök, M. \& Perälä-Littunen, S. (2008). 'Children Need Their Parents More Than a Pizza in the Fridge!': Parental responsibility in a Finnish newspaper. Childhood, 15(1): 7488. https://10.1177/0907568207086836

Burman, E. (2012). Disciplines for and against Psychology. Universitas Psychologica, 11(2), 645-662. Recuperado de http://revistas.javeriana.edu.co/index.p hp/revPsycho/article/view/1464

Burman, E. (1998). La Deconstrucción de la Psicología Evolutiva. Madrid: Editorial Antonio Machado Libros.

Cerri, Ch. \& Alamillo-Martínez, L. (2012). La organización de los cuidados, más allá de la dicotomía entre esfera pública y esfera privada. Gazeta de Antropología, 28(2), 1-22. Recuperado de http:/hdl.han dle.net/10481/23793

Chávez, P. (2015). La palabra de los niños como discurso social: análisis crítico del discurso de niños y niñas de distintos estratos socioeconómicos de Santiago de Chile sobre la relación entre padres/madres e hijos(as) [tesis de Doctorado]. Universidad de Chile, Santiago, Chile.

Cockburn, T. (2005). Children and the feminist ethic of care. Childhood, 12(1), 71-89. http s://doi.org/10.1177/0907568205049893

Comas d'Argemir, D. (2014). La crisis de los cuidados como crisis de reproducción social. Las políticas públicas y más allá. En Periferias, fronteras y diálogos. Actas del XIII Congreso de Antropología de la FAAEE (pp.
329-349). España: Publicacions Universitat Rovira I Virgili.

Fairclough, N. (1995). Critical discourse analysis. The critical study of language. London: Longman.

Fairclough, N. (2003). Analysing discourse. Textual analysis for social research. London: Routledge.

Giberti, E. (1997). La niñez y el hacer política. En E. Giberti (Comp), Políticas y niñez (pp. 23-113). Buenos Aires: Losada.

Gilligan, C. (1982). In a different voice: psychological theory and women's development. Cambridge: Harvard University press.

Katz, E. (2013). Domestic Violence, Children's Agency and Mother-Child Relationships: Towards a More Advanced Model. Children E3 Society, 29(1), 69-79. https://doi.org/10.1 111/chso. 12023

Lee, E., Bristow, J., Faircloth Ch. \& Macvarish J. (2014). Parenting Culture Studies. London: Palgrave Macmillan.

Legarreta, M. (2006). Sobre el trabajo y los trabajos (o las polisemias del trabajo): Reflexiones desde una perspectiva feminista. En Laboratorio Feminista (Ed), Transformaciones del trabajo desde una perspectiva feminista: producción, reproducción, deseo, consumo (pp.217-232). Madrid: Tierra de Nadie ediciones.

Luttrell, W. (2007). Children's Counternarratives of Care: Towards Educational Justice. Children EF Society, 27(4), 295-308. https://doi.org/10.1111/chso.12033

Luttrell, W. (2012). Making Boys' care worlds visible. Thymos, 6(2), 186-202. https://doi. org/10.3149/thy.0602.186

Macri, M., Ford, M., Berliner, C. \& Molteni, M. J. (2005). El trabajo Infantil no es Juego. Estudios e Investigaciones sobre trabajo infanto-adolescente en Argentina (1900-2003). Buenos Aires: Editorial Stella \& Ediciones La Crujía.

Mayall, B. (2002). Towards a sociology for childhood: Thinking from Children's Lives Buckingham: Open University Press. 
Pérez, A. (2006). Amenaza tormenta: La crisis de los cuidados y la reorganización del sistema económico. Revista de Economía Crítica, (5), 7-37. Recuperado de http:// observatoridesc.org/sites/default/files/lame nazatormenta.pdf

Prout, A. \& James, A. (1997). A new paradigm for the sociology of childhood?. Provenance, promise and problems. En A. James \& A. Prout (Eds), Constructing and reconstructing childhood. Contemporary issues in the sociological study of childhood (pp.

7-33). London: RoutledgeFalmer.

Qvortrup, J. (1992). El niño como sujeto y objeto: ideas sobre el programa de infancia en el Centro europeo de Viena. Infancia y sociedad, (15), 169-186.

Qvortrup, J., Corsaro, W. \& Honig, M.S. (Eds) (2009). The Palgrave Handbook of Childhood Studies. Inglaterra: Palgrave Macmillan. viii Si bien los niños varones de este estrato también participan, aunque en menor medida, en la realización de estas tareas, ellos significan esta participación como una forma de 'ayudar' a las mujeres en el cuidado y mantención del hogar, no como expresión de su posición de género en la familia.

* Artículo de investigación.

\section{Notas}

i Paraíso perdido, sabiduría o libertad originaria, tiempo de inocencia e ignorancia, de peligro y vulnerabilidad, entre otras.

ii La palabra "hijidad" es un neologismo que utilizado en ausencia de una palabra en español que permite hacer referencia a las prácticas asociadas a la condición de hijo(a).

iii Por restricciones de espacio, el presente análisis se centra básicamente en las similitudes de los discursos de los distintos grupos.

iv Una "buena" infancia que aparece asociada a los ideales convencionales modernos de una infancia protegida, dependiente, separada radicalmente del mundo adulto, caracterizada por el predominio de la fantasía, el juego, etc.

v Mundo adulto asociado al trabajo, responsabilidades, esfuerzo y sacrificio permanentes.

vi Dos mil pesos chilenos.

vii Esta noción refiere a "las historias que las personas viven y cuentan y que ofrecen resistencia, ya sea implícita o explícitamente, a las narrativas culturales dominantes" (Luttrell, 2007, p. 295). 